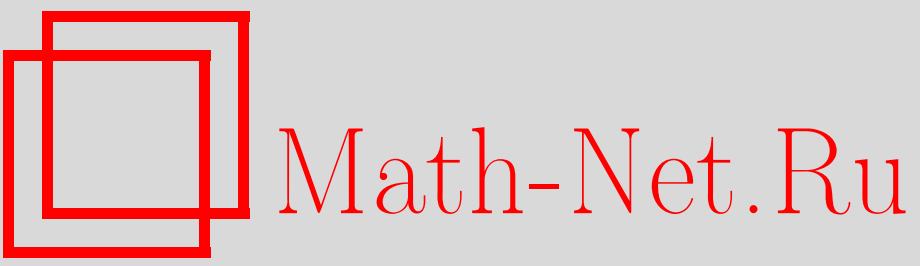

М. Яворский, Экспоненциально сужающийся джозефсоновский контакт: некоторые аналитические результаты, ТМФ, 2005, том 144, номер 2, 348-353

DOI: https://doi.org/10.4213/tmf1860

Использование Общероссийского математического портала Math-Net.Ru подразумевает, что вы прочитали и согласны с пользовательским соглашением

http://www.mathnet.ru/rus/agreement

Параметры загрузки:

IP : 54.162 .85 .209

26 апреля 2023 г., 14:42:58 
ТЕОРЕТИЧЕСКАЯ

И МАТЕМАТИЧЕСКАЯ

ФИЗИКА

Том 144, № 2

август, 2005

(C) 2005 г.

М. Яворский*

\title{
ЭКСПОНЕНЦИАЛЬНО СУЖАЮШИЙСЯ ДЖОЗЕФСОНОВСКИЙ КОНТАКТ: НЕКОТОРЫЕ АНАЛИТИЧЕСКИЕ РЕЗУЛЬТАТЫ
}

\begin{abstract}
Динамические свойства экспоненциально сужающегося джозефсоновского контакта исследуются на основе простой одномерной модели, описывающей возмушенное (почти интегрируемое) уравнение синус-Гордон. Приближенное аналитическое решение основано на линеаризации вблизи быстроосциллирующего фона. Аналитические результаты сравниваются с прямым численным моделированием распределения магнитного поля в контакте.
\end{abstract}

Ключевые слова: джозефсоновский контакт, уравнение синус-Гордон.

\section{1. ВВЕДЕНИЕ}

Экспоненциально сужающиеся джозефсоновские контакты в последнее время привлекают к себе значительное внимание ввиду возможных применений в mm-волновой сверхпроводящей электронике [1]. Такая неравномерная структура позволяет добиться некоторых преимушеств по сравнению со стандартным прямоугольным контактом, а именно, получить меньшую ширину линии, бо́льшую выходную мошность и лучшее согласование сопротивлений.

До сих пор джозефсоновские контакты экспоненциальной формы исследовались численно и экспериментально. Целью данной работы является представить приближенное аналитическое решение соответствуюшего возмущенного уравнения синус-Гордон.

Вещественная двумерная структура сначала редуцируется к одномерной модели, а затем приближенное решение находится в форме малых гармонических колебаний, наложенных на сильно нелинейный, быстроосциллирующий фон. Определив решение, удовлетворяющее подходящим граничным условиям, мы можем найти некоторые физически адекватные величины, например, распределение магнитного поля в контакте.

*Institute of Physics, Polish Academy of Sciences, Al. Lotników, 32/46, 02-668, Warszawa, Poland. E-mail: jawor@ifpan.edu.pl 


\section{2. ДВУМЕРНЫЙ ЭКСПОНЕНЦИАЛЬНО СУЖАЮШИЙСЯ КОНТАКТ И ОДНОМЕРНАЯ МОДЕЛЬ}

Двумерньй экспоненциально сужающийся джозефсоновский контакт, помешенный во внешнее однородное магнитное поле, показан на рис. 1. Контакт можно описать двумерным модифицированным уравнением синус-Гордон

$$
\Delta \phi-\phi_{t t}-\alpha \phi_{t}=\sin \phi,
$$

где $\phi$ - джозефсоновская фаза, а $\alpha$ - множитель, описывающий затухание из-за нормальных электронов. Уравнение (1) записано в безразмерных единицах, т.е. пространственные координаты нормированы на джозефсоновскую глубину проникновения $\lambda_{J}$, а временна́я координата - на обратную плазменную частоту $\omega_{0}^{-1}$.

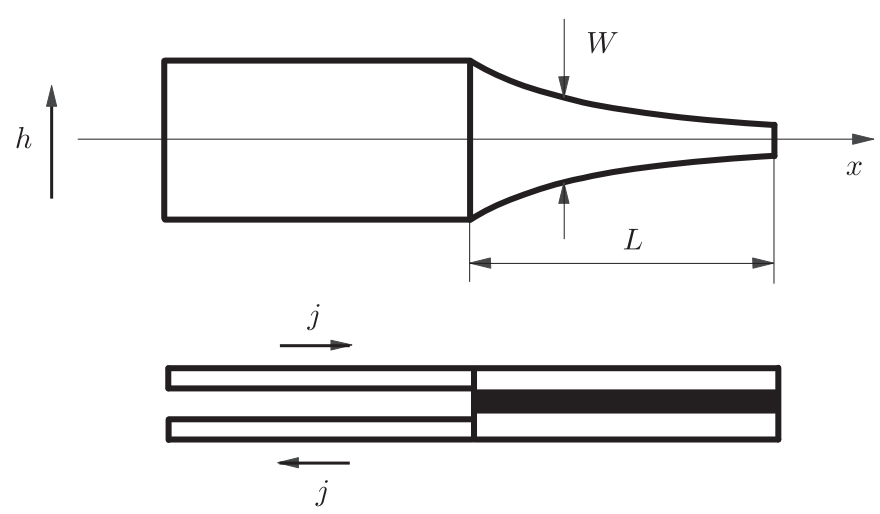

Рис. 1. Экспоненциально сужающийся джозефсоновский контакт. $W$ - ширина контакта,

$L$ - длина контакта, $h$ - внешнее магнитное поле, $j$ - постоянный ток смешения.

Будем предполагать, что ширина контакта экспоненциально зависит от $x$, т.е. $W(x)=$ $W_{0} \exp (-\lambda x)$. Если контакт является узким $(W(x) \ll 1)$ и его ширина медленно меняется (величина $\lambda$ мала), то можно редуцировать двумерное уравнение синус-Гордон (1) к одномерному почти интегрируемому уравнению [1]:

$$
\phi_{x x}-\phi_{t t}-\alpha \phi_{t}=\sin \phi+\lambda \phi_{x}-\lambda h .
$$

Согласно уравнениям Джозеф̆сона [2] функция $\phi_{t}$ пропорциональна электрическому полю, а $\phi_{x}$ - магнитному полю в контакте. Таким образом, для тока смешения $j$, приложенного к левому концу контакта, граничные условия можно записать в виде

$$
\phi_{x}(-L / 2)=h-j, \quad \phi_{x}(L / 2)=h,
$$

где $h$ - внешнее однородное магнитное поле, а $L$ - длина контакта.

Поскольку наша цель состоит в том, чтобы дать аналитическое описание реальной экспериментальной ситуации, мы ограничимся строго периодическими по времени решениями для стационарных состояний, в которых омические потери сбалансированы энергией, доставляемой через края контакта. 


\section{3. ПРИБЛИЖЕННОЕ АНАЛИТИЧЕСКОЕ РЕШЕНИЕ}

Для длинного контакта $(L \gg 1)$ и достаточно сильного внешнего поля $h$ мы ишем решение в виде "flux-flow" моды, т.е. в виде плотного пакета солитонов, движущихся в одном направлении на быстроврашающемся фоне [3], [4],

$$
\phi=\phi_{0}+\psi
$$

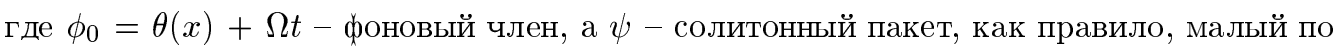
величине и гармонический по $\Omega t$.

Идея врашаюшегося фона следует непосредственно из “обратного” преобразования, примененного к точному периодическому решению стандартного уравнения синус-Гордон в пределе плотного пакета солитонов [5]. Аналогичньй подход, основанный, однако, на других аргументах, был предложен в работах [3], [6].

Используя анзац (4), можно рассмотреть различные временны́е масштабы и найти по отдельности две части уравнения (2): не зависяшую от времени и гармоническую по $\Omega t$. Соответственно, граничные условия можно записать отдельно для $\phi_{0}$ :

$$
\phi_{0, x}(-L / 2)=h-j, \quad \phi_{0, x}(L / 2)=h,
$$

и $\psi$ :

$$
\psi_{x}(-L / 2)=0, \quad \psi_{x}(L / 2)=0
$$

3.1. Не зависящие от времени члены. Подставляя уравнение (4) в уравнение (2) и собирая не зависящие от времени члены, получаем уравнение

$$
\theta_{x x}-\alpha \Omega=\lambda \theta_{x}-\lambda h
$$

Строго говоря, член $\psi \cos \phi_{0}$ также дает вклад в не зависящую от времени часть уравнения. Однако эта поправка обычно мала и будет учтена в дальнейшем в серии последовательных приближений.

Используя стандартные методы, из уравнения (7) находим

$$
\theta=\left(h-\frac{\alpha \Omega}{\lambda}\right) x+\frac{\alpha \Omega}{\lambda^{2}} e^{\lambda(x-L / 2)},
$$

причем граничные условия дают

$$
j=\frac{\alpha \Omega}{\lambda}\left(1-e^{-\lambda L}\right)
$$

Заметим, что усредненное по времени значение $\phi_{t}$ равняется $\Omega$ и пропорционально постоянному напряжению на контакте. Таким образом, уравнение (9) можно интерпретировать как омическую цепь, что хорошо согласуется с экспериментальными результатами [3]. 
3.2. Линеаризованное уравнение. Для гармонических по $\Omega t$ членов получаем соотношение

$$
\psi_{x x}-\psi_{t t}-\alpha \psi_{t}-\lambda \psi_{x}=\sin \phi_{0},
$$

где член $\psi \cos \phi_{0}$ опушен, поскольку он не дает вклада на частоте $\Omega$.

Используя комплексные обозначения

$$
\psi(x, t)=\operatorname{Im}[\tilde{\psi}(x, t)]=\operatorname{Im}\left[\hat{\psi}(x) e^{i \Omega t}\right]
$$

можно преобразовать уравнение (10) к виду

$$
\hat{\psi}_{x x}-\lambda \hat{\psi}_{x}+\beta^{2} \hat{\psi}=e^{i \theta},
$$

где $\beta^{2}=\Omega^{2}-i \alpha \Omega$.

Обшее решение уравнения (12) имеет вид

$$
\hat{\psi}=\frac{1}{2 i \beta}\left[e^{r_{1} x} F_{1}(x)-e^{r_{2} x} F_{2}(x)\right]+A e^{r_{1} x}+B e^{r_{2} x},
$$

где $r_{1,2}=\lambda / 2 \pm i \beta$ и

$$
F_{n}(x)=\int_{-L / 2}^{x} e^{i \theta-r_{n} \xi} d \xi, \quad n=1,2 .
$$

Первый член в уравнении (13) представляет собой "flux-flow" моду, т.е. плотньй пакет солитонов, а два последних члена соответствуют плазменным волнам, распространяющимся в противоположных направлениях с критической скоростью $\Omega / \beta \simeq 1$.

Постоянные $A$ и $B$ можно определить из граничных условий (6):

$$
\begin{aligned}
A & =\frac{r_{1} F_{1}(L / 2) e^{i \beta L}-r_{2} F_{2}(L / 2)}{4 r_{1} \beta \sin (\beta L)} \\
B & =\frac{-r_{1} F_{1}(L / 2)+r_{2} F_{2}(L / 2) e^{-i \beta L}}{4 r_{2} \beta \sin (\beta L)} .
\end{aligned}
$$

Подставляя $A$ и $B$ в уравнение (13) и далее в уравнение (4), получаем приближенное решение уравнения (2), удовлетворяюшее граничным условиям (3).

Определив решение $\hat{\psi}$, можно вернуться к не зависящей от времени части уравнения (2) и учесть поправку, возникающую в связи с наличием члена $\psi \cos \phi_{0}$. Легко проверить, что соответствуюшая поправка имеет вид $\operatorname{Im}\left[\hat{\psi} e^{-i \theta}\right] / 2$. Таким образом, снова решая уравнение $(7)$ с добавлением указанной поправки в правую часть, получаем новое решение $\theta$, которое, в свою очередь, порождает новое решение $\hat{\psi}$. Численные проверки показывают, что такой итерационный процесс быстро сходится, и при характерных параметрах контакта достаточно выполнить лишь несколько итераций для получения самосогласованного решения.

Здесь следует упомянуть, что член $\psi \cos \phi_{0}$ порождает также вторую гармонику по $\Omega t$. Однако спектральный анализ численных данных, как и некоторые теоретические оценки, показывают, что высшие гармоники пренебрежимо малы и практически не влияют на решение при характерных значениях параметров контакта. 

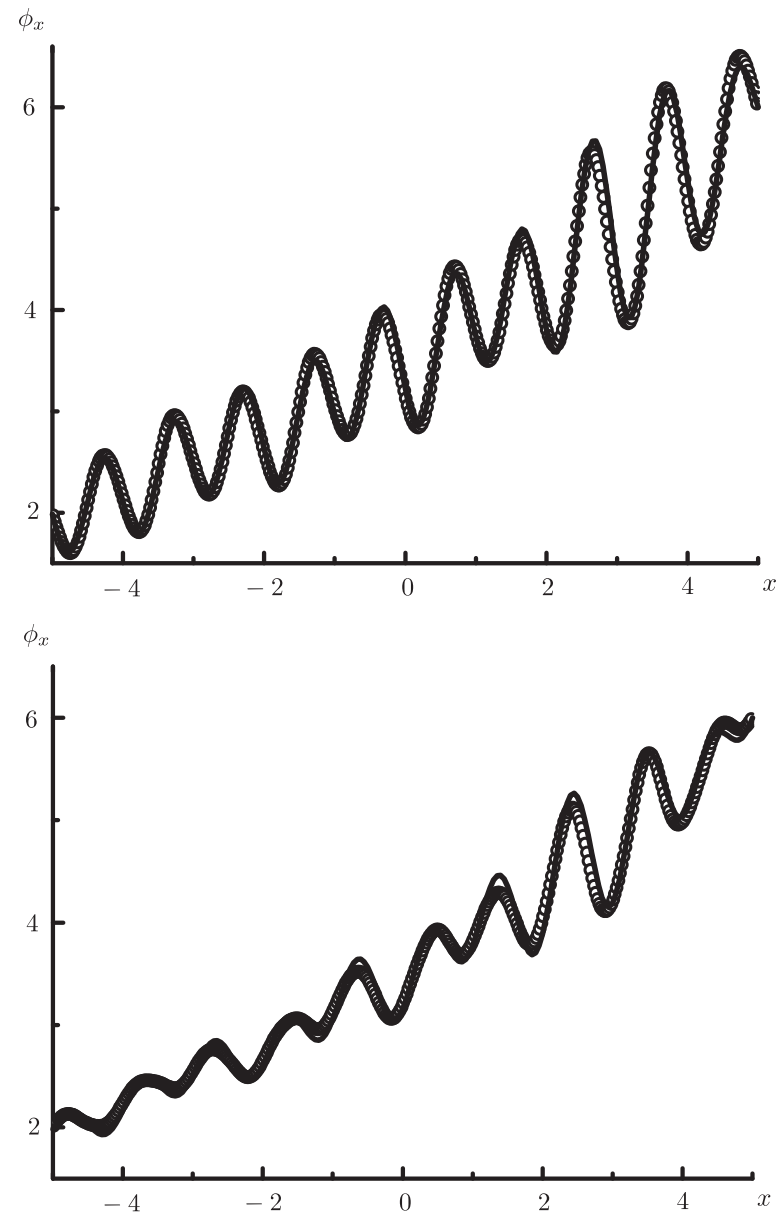

Рис. 2. Распределение магнитного поля в длинном контакте в два различных момента времени, полученное аналитически (сплошная линия) и численно (полые окружности) при $L=10, h=6, \alpha=0.1, \lambda=0.1, j=4$.

\section{4. РЕЗУЛЬТАТЫ И ОБСУЖДЕНИЕ}

В данном разделе приведеные вьше аналитические результаты сравниваются с прямым численным моделированием, основанным на неявной конечно-разностной схеме. Примеры распределения магнитного поля в длинном контакте $(L=10)$ в два произвольно выбранных момента времени приведены на рис. 2. Для тока смещения выбрано значение $j=4$, соответствуюшее $\Omega \simeq 6.29$. Для остальных параметров предполагаются характерные экспериментальные значения: $h=6, \alpha=0.1, \lambda=0.1$. Сплошная линия представляет собой приближенное аналитическое решение уравнения $(2)$, изображенное как $\phi_{x}(x)$ при фиксированном $t$. Полье окружности показывают результаты численного моделирования для дискретного набора значений $x$.

На обоих рисунках легко видеть, что солитонный пакет в самом деле является плот- 
ным, формируя в действительности колебания с малой амплитудой, сильно модулированные за счет интерференции с плазменными волнами (см. два последних члена в уравнении (13)). Осциллируюшая часть решения наложена на фон $\phi_{0}$, производная которого по $x$ не зависит от времени, но растет экспоненциально вместе с $x$ (см. уравнение (8)), что позволяет удовлетворить асимметричные граничные условия (3). Согласие между аналитическими и численными результатами оказывается превосходным, и это означает, что выведенное выше приближенное аналитическое решение, несмотря на свою простоту, правильно воспроизводит все детали точного решения.

\section{5. ЗАКЛЮЧЕНИЕ}

В данной работе представлена простая модель, позволяюшая вывести приближенное аналитическое решение для случая двумерного экспоненциально сужаюшегося джозефсоновского контакта. Решение дано в виде быстроврашаюшегося зависяшего от $x$ фонового члена, сопровождаемого периодическими по времени квазилинейными членами.

Сравнение аналитических результатов и численных данных показывает, что данная модель удовлетворительно описывает динамические свойства джозефсоновских контактов экспоненциальной формы. В частности, правильно воспроизводятся все детали распределения магнитного поля в контакте.

Как отмечено выше, представленый здесь метод позволяет также определить некоторые макроскопические, непосредственно измеряемые величины, такие как полный ток смешения и постоянное напряжение на контакте. Такие более экспериментально-ориентированные результаты, равно как и другие физические аспекты джозефсоновских контактов экспоненциальной формы, не укладываются в рамки данной статьи и будут рассмотрены отдельно.

Благодарности. Работа былаподдержана со стороны KBN, грант № 2P03В 09722.

\section{Список литературы}

[1] S. Pagano, C. Nappi, R. Cristiano et al. A long Josephson function-vased device for particle detection. In: Proc. of Conf. on Nonlinear Superconducting Devices and High $T_{c}$ Materials (Capri, Italy, 8-13 October, 1994). Eds. R. D. Parmentier, R.F. Pedersen. Singapore: World Scientific, 1995. P. 437; A. Benabdallah, J. G. Caputo, A. C. Scott. Phys. Rev. B. 1996. V. 54. P. 16139; G. Carapella, N. Martuciello, G. Costabile. Phys. Rev. B. 2002. V. 66. P. 134531.

[2] A. Barone, G. Paterno. Physics and Applications of the Josephson Effect. N.Y.: Wiley, 1982.

[3] M. Cirillo, N. Grønbech-Jensen, M.R. Samuelsen, M. Salerno, G. Verona Rinati. Phys. Rev. B. 1998. V. 58. P. 12377.

[4] M. Jaworski. Phys. Rev. B. 1999. V. 60. P. 7484; M. Jaworski. Supercond. Sci. Technol. 2004. V. 17. P. 327.

[5] M. Jaworski. Phys. Lett. A. 1998. V. 244. P. 97.

[6] M. Salerno, M. Samuelsen. Phys. Rev. B. 1999. V. 59. P. 14653. 